\title{
Interfacial Electron Transfer and Ion Solvation in the Solid Electrolyte Interphase
}

Jeongmin Kim, Brett Savoie, Thomas Miller

Submitted date: 16/12/2020 - Posted date: 22/12/2020

Licence: CC BY-NC-ND 4.0

Citation information: Kim, Jeongmin; Savoie, Brett; Miller, Thomas (2020): Interfacial Electron Transfer and Ion Solvation in the Solid Electrolyte Interphase. ChemRxiv. Preprint.

https://doi.org/10.26434/chemrxiv.13385186.v1

As a chemically and structurally well-defined model for redox processes in the solid electrolyte interphase of battery electrodes, we investigate electron transfer to lithium ions at the interface between a platinum metal anode and a solid polymer electrolyte. Studied electrolytes include LiTFSI (lithium bis(trifluoromethane)sulfonimide) salts in polyethylene oxide and poly(diethylene oxide-alt-oxymethylene), as well as in the as- sociated liquid electrolytes 1,2-dimethoxyethane and tetraglyme.

File list (2) 


\title{
Interfacial Electron Transfer and Ion Solvation in the Solid Electrolyte Interphase
}

\author{
Jeongmin Kim, ${ }^{\dagger}$ Brett M. Savoie, ${ }^{\ddagger}$ and Thomas F. Miller $\mathrm{III}^{*, \dagger}$ \\ $\dagger$ Division of Chemistry and Chemical Engineering, California Institute of Technology, \\ Pasadena, California, USA \\ $\ddagger$ School of Chemical Engineering, Purdue University, West Lafayette, Indiana, USA \\ E-mail: tfm@caltech.edu
}

\begin{abstract}
As a chemically and structurally well-defined model for redox processes in the solid electrolyte interphase of battery electrodes, we investigate electron transfer to lithium ions at the interface between a platinum metal anode and a solid polymer electrolyte. Studied electrolytes include LiTFSI (lithium bis(trifluoromethane)sulfonimide) salts in polyethylene oxide and poly(diethylene oxide-alt-oxymethylene), as well as in the associated liquid electrolytes 1,2-dimethoxyethane and tetraglyme. Atomic-resolution simulations are performed with constant-potential polarizable electrodes to characterize interfacial electron-transfer kinetics, including lithium-ion solvation structures and solvent reorganization effects as a function of applied electrode potential. The linearresponse assumptions of the Marcus theory for electron transfer are found to be robust in these systems, yet ion-solvation behavior at the anode interface is strikingly dependent on chain connectivity, solvation environment, and the magnitude of the applied electrode potential, resulting in very different electron-transfer kinetics for lithium electroreduction.
\end{abstract}




\section{Introduction}

Interfacial stability is essential for cycling performance and longevity of rechargeable batteries. $^{1-9}$ For example, lithium-ion battery (LIB) and lithum-metal battery (LMB) interfaces involve various competitive electrochemical reactions, including the electroreduction of non-aqueous electrolytes and salts. ${ }^{2,3,10}$ These processes result in a structurally and chemically heterogeneous thin film on the electrode surface called the solid electrolyte interphase (SEI), ${ }^{11-18}$ which is a complicated mix of organic, inorganic, and polymeric components. A functionally useful SEI passivates the electrode, conducting ions yet providing electronic insulation to mitigate detrimental electrolyte degradation that leads to battery aging and failure.

Polymeric components in the SEI have been shown to play a role in electrode passivation, including polyether, ${ }^{19-22}$ polyvinylene carbonate, ${ }^{23,24}$ polycarbonate, ${ }^{25,26}$ and polyolefin species. ${ }^{27}$ These systems present diverse environments for ion solvation that may be expected to substantially effect the mechanisms and kinetics of interfacial electron transfer; ${ }^{28-37}$ for example, recent work in molecular liquids has shown that hydrogen bond networks and ion-solvation properties ${ }^{38-41}$ lead to non-trivial interfacial phenomena such as heterogenous charge separation or collective water exchange. ${ }^{41,42}$ However, relatively little is known about ion solvation and electron transfer (ET) in the SEI, which is complicated by the intrinsic heterogeneity and complexity of this material. To address this challenge, we introduce polymer melts as well-defined chemical models for the SEI, enabling mechanistic investigation of ET at the polymer/electrode interface as a proxy for the realistic battery electrode.

In this work, molecular dynamics (MD) simulations for atomic-resolution models are performed with constant-potential polarizable electrodes to study factors that govern lithium electroreduction at a polymer/metal interface. Linear ether homopolymer electrolytes are considered as chemically and structurally well-defined models for the SEI, including poly(ethylene oxide) (PEO) and poly(diethylene oxide-alt-oxymethylene), P(2EO-MO). Previous work indicates that polyethers are formed in the SEI via either in-situ or ex-situ polymerization 
of 1,3-dioxolane (DOL), leading to enhanced cycling stability. ${ }^{19-21}$ Associated liquid ether electrolytes, 1,2-dimethoxyethane (DME) and tetraglyme (G4), are also investigated to explore the degree to which polymerization alters the local monomer interactions with regard to properties that are relevant for electron transfer.
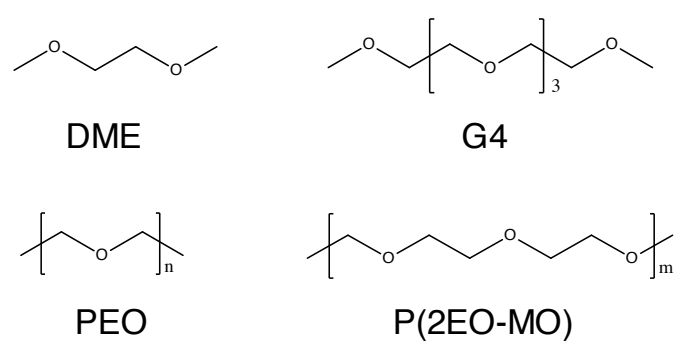

Figure 1: Chemical structures of the ethereal molecular electrolytes (DME and G4) and polymer electrolytes (PEO and $\mathrm{P}(2 \mathrm{EO}-\mathrm{MO})$ ).

\section{Methods and Calculation Details}

We consider a model system that is comprised of two metal electrodes and an electrolyte of LiTFSI salt in either polymer or molecular liquid solvent (Fig. 1). The electrodes are modeled using the pristine (111) surface of face-centered cubic (FCC) platinum (Fig. 2). Below, we describe the computational details of the interaction potentials, MD simulations, calculated vertical ionization energies, calculated free energy curves associated with an electrochemical ET, and calculated normalized local density of ions and electrolytes. All simulations are conducted using the LAMMPS simulation package, ${ }^{43}$ and all force field parameters used in this study are provided in Ref 44 .

\subsection{Force field details and the constant-potential method}

The TraPPE-UA force field, ${ }^{44-46}$ a non-polarizable and united-atom model, is used to describe the potential energy functions of the polymers and molecular liquids. The LiTFSI salt is described using the non-polarizable force field of Lopez et al. ${ }^{47}$ Among all atoms of elec- 
(A)

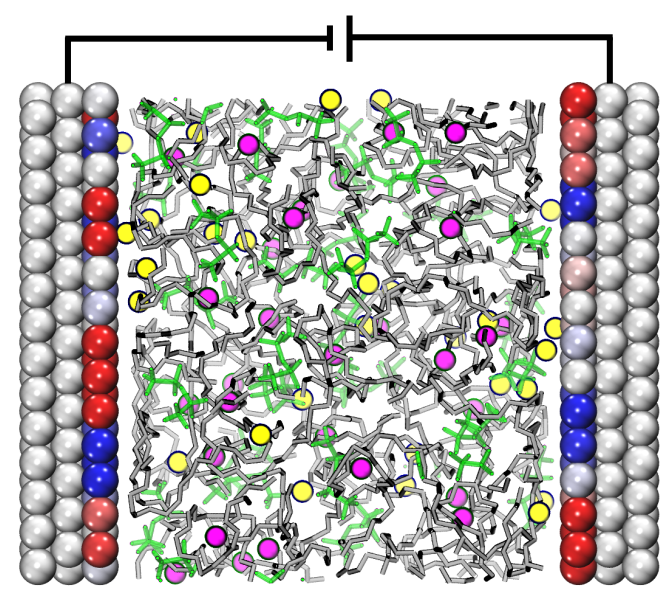

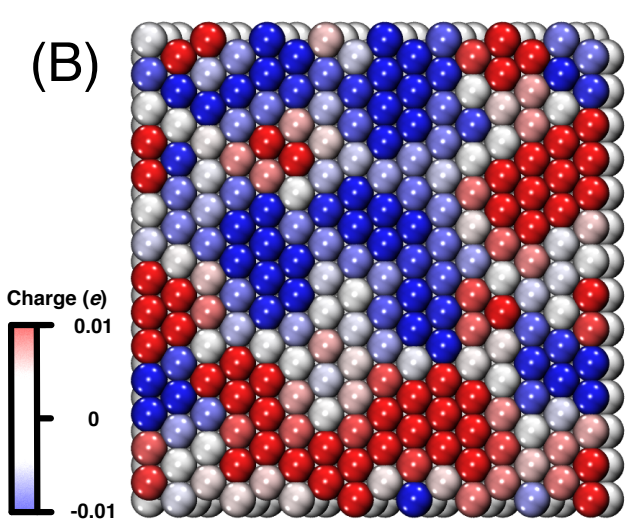

Figure 2: (A) A simulation snapshot including PEO, $\mathrm{Li}^{+}$ions, $\mathrm{TFSI}^{-}$ions, $\mathrm{Li}^{0}$ atoms, and model electrodes. The grey strand represents a single PEO chain. Purple spheres represent $\mathrm{Li}^{+}$ions while yellow ones do neutral $\mathrm{Li}^{0}$ atoms. TFSI ${ }^{-}$ions are drawn in green. The two slabs are pristine, polarizable model electrodes held with the bias potential $\Delta \Psi=0 \mathrm{~V}$. Color for the electrode atoms is associated with their induced charges between -0.01 (blue) and +0.01 (red). (B) Electrode-charge polarization on the innermost layer of the anode in (A).

trolytes and salts, we employ the standard Lorentz-Berthelot mixing rule for the TraPPE-UA force field, $\sigma_{i j}=0.5\left(\sigma_{i}+\sigma_{j}\right)$ and $\epsilon_{i j}=\sqrt{\epsilon_{i} \epsilon_{j}}$, where $\sigma_{i}$ and $\epsilon_{i}$ correspond to the usual LJ parameters for atom $i$.

For the platinum electrodes, the constant potential method (CPM) is employed to provide a polarizable description of the electrostatic interactions and to allow for simulation at constant applied potential. ${ }^{48-50}$ This method accounts for the effect of image-charge formation in the electrode in response to charges in the electrolyte (Fig. 2). Each electrode atom carries an atom-centered spherical Gaussian charge distribution with fixed width $(\eta=1.979$ $\AA^{-1}$ ) and a time-dependent amplitude, $A_{i}(t)$ that is determined as a function of the position of the other atomic charges in the system,

$$
Q_{i}(\vec{r}, t)=A_{i}(t)\left(\frac{\eta^{2}}{\pi}\right)^{3 / 2} \exp \left[-\eta^{2}\left(\vec{r}-\vec{R}_{i}\right)\right]
$$

where $\vec{R}_{i}$ is the fixed position of an $i^{\text {th }}$ electrode atom. We employ the version of the CPM developed in Ref.51, which employs matrix inversion at each timestep to determine the charge 
polarization on the electrode atoms. The metal slabs include three layers of atoms, which has been found to provide a sufficiently converged description of the electrode polarization. ${ }^{48,49}$ The cathode layers are held at a positive electrode-potential, $\Psi^{+}$and the anode layers are held at a negative electrode-potential, $\Psi^{-}\left(=-\Psi^{+}\right)$. The bias potential is $\Delta \Psi=\Psi^{+}-\Psi^{-}$. Electrode potential in this study is in the unit of volt.

For the non-Coulomb interactions involving the electrode atoms, we fit the platinum force field of Heinz et al. ${ }^{52}$ to the LJ interaction form, yielding the platinum LJ parameters $\epsilon=7.8$ $\mathrm{kcal} / \mathrm{mol}$ and $\sigma=2.534 \AA$. As prescribed by this force field, we employ the geometrical mixing rule for the LJ interactions between the platinum and electrolyte atoms. However, we reduce the LJ interactions between the platinum and electrolyte atoms by half throughout this study, such that $\epsilon_{i j}=0.5 \sqrt{\epsilon_{i} \epsilon_{j}}$ to approximately match the adsorption energy of acetonitrile on platinum. ${ }^{53}$ Finally, to avoid rare, unphysical penetration of the lithium ions into the anode layers during the simulations, we employ the additional short-ranged repulsive potential

$$
\begin{aligned}
U_{w}(z) & =4 \epsilon_{w}\left[\left(\frac{\sigma_{w}}{z-z_{w}}\right)^{12}-\left(\frac{\sigma_{w}}{z-z_{w}}\right)^{6}\right] \text { if } z<z_{c} \\
& =0 \text { otherwise }
\end{aligned}
$$

where $\epsilon_{w}=7.9597 \mathrm{kcal} / \mathrm{mol}=10 R \mathrm{~T}$ with the gas constant $R, \sigma_{w}=2.575 \AA, z_{w}=z_{c}-\sigma_{w}$, and $z_{c}$ is variable with the solvents, which is position of the atoms in the exterior layer of metal atoms on the electrode along the perpendicular component. This steep repulsive potential has no effect on ion solvation.

\subsection{MD simulations}

For the polymer-electrolyte simulations, we employ a single linear polymer chain of length 1000 and 333 units for $\mathrm{PEO}$ and $\mathrm{P}(2 \mathrm{EO}-\mathrm{MO})$, respectively. So, multi-chain effect in the polymers is excluded. For the liquid-electrolyte simulations, 500 DME molecules or $200 \mathrm{G} 4$ ones are employed. Chemical structures of all four solvents are displayed in Fig 1. For 
LiTFSI salt, we keep a ratio of a lithium ion to chemical moieties of each solvent the same such that $[\mathrm{EO}]:\left[\mathrm{Li}^{+}\right]=15: 1$, where $[\mathrm{EO}]$, and $\left[\mathrm{Li}^{+}\right]$are the number density of ether oxygen for the electrolytes, and $\mathrm{Li}^{+}$ions, respectively.

In all cases during both equilibration and production runs, the MD trajectories are integrated using the velocity-Verlet methods with a timestep of $1 \mathrm{fs}$. Both LJ and Coulomb interactions are cut at $14 \AA$, and particle-particle particle-mesh Ewald summation is used to compute Coulomb interactions beyond the cutoff distance. Periodic boundary conditions (PBCs) are applied along $x y$ directions only, unless otherwise noted. Moreover, to prevent the long-range contribution of Coulomb interaction along $z$ direction, the vacuum region of the equal size to the simulation cell is introduced on both sides along the transverse direction. ${ }^{54}$ The Nosé-Hoover thermostat (100 fs relaxation) and the Nosé-Hoover barostat (1000 fs relaxation) along $x y$ directions are applied in all simulations to control the temperature $(400 \mathrm{~K})$ and the pressure $(1 \mathrm{~atm})$, unless otherwise noted.

Equilibration of both polymer and molecular electrolytes involves four steps. The first step follows a protocol of Ref 44 which involves steepest descent energy minimization, Langevin dynamics at elevated temperature, and annealing process. This step takes at least $10 \mathrm{~ns}$ with PBCs and the barostat along all three directions. Secondly, electrode atoms are introduced at both end of the simulation cell along $\mathrm{z}$ direction without any overlaps. Lateral dimensions of the cell are slightly modified to meet the lattice periodicity of 111 surface of FCC electrodes. Then, LiTFSI salt is randomly placed in the simulation cell without overlaps with electrode atoms. Steepest descent energy minimization is employed with frozen electrode atoms in space to avoid unduly high forces due to the newly added salt atoms. Thirdly, $z$ position of the electrode atoms is adjusted in order to remove the undesirable pressure effect across the cell. Each of the electrodes moves as a rigid body according to the forces including the constant force to meet the desired pressure (1 atm) along z direction, and average force from electrolytes. This step takes at least $10 \mathrm{~ns}$. After equilibrated, the transverse box length fluctuates with time following a Gaussian distribution. 
Electrode atoms are fixed in space in accordance to the mean of the Gaussian distribution. Lastly, neutral lithium atoms are added into the simulation cell at random places without overlaps with the electrode atoms. After steepest descent energy minimization, systems are equilibrated with the CPM turned on at each electrode potential during at least 5 ns for molecular-electrolyte or 10 ns for polymer-electrolyte systems.

\subsection{Vertical ionization energy calculations}

For the characterization of electrochemical ET, the vertical ionization energy $(\Delta E)$ is computed to provide a reaction coordinate for an electrochemical ET reaction between a lithium species and an anode. ${ }^{55}$ For the half-reaction associated with the oxidation of $\mathrm{Li}^{0}, \Delta E=$

$\delta E^{\text {anode }}+I+\delta q W$, where $\delta E^{\text {anode }}$ is the difference in total potential energy, $\delta q$ is the amount of transferred charge during the half-reaction under a frozen solvent configuration, $I$ is ionization energy of lithium, and $W$ is work function of a metal electrode. Similarly, for the half-reaction associated with the reduction of $\mathrm{Li}^{+}, \Delta E=-\delta E^{\text {anode }}+I+\delta q W$. The term, $\delta E^{\text {anode }}$ depends on the distance of lithium species form the anode, whereas $I+\delta q W$ does not. The term, $I+\delta q W$ is a constant with respect to the lithium position and electrode potential whose value ensures a criterion that free energy curves associated with the ET cross each other at $\Delta E=0$ is satisfied. ${ }^{56}$

To calculate $\delta E^{\text {anode }}$ in the presence of the constant-potential electrodes, the approach of Ref. 56 is employed for a given configuration sampled every 0.1 ns. Two terms contribute to $\delta E^{\text {anode }}: \delta E^{\text {anode }}=\delta E+\delta E^{\text {elec }}$, where $\delta E$ is the difference in total potential energy upon an $\mathrm{ET}$ and $\delta E^{\mathrm{elec}}$ is a correction term. The correction term should be added due to the fact that both electrodes participate in the ET reaction in our simulations on the contrary to the actual experimental situation where only one of them is involved. For a single lithium species, its identity is swapped under a frozen solvent configuration: $\mathrm{Li}^{0} \rightarrow \mathrm{Li}^{+}$for oxidation or $\mathrm{Li}^{+} \rightarrow \mathrm{Li}^{0}$ for reduction. The identity swap for the lithium species is performed by turning on (off) Coulomb interaction with all other atoms for the oxidation (the reduction) 
with the same LJ interaction parameters, so the difference in total potential energy should be the same with the difference in total Coulomb energy. Further, the effect of electrode-charge polarization should be included, whose response is treated adiabatically. After the electrodecharge polarization is recalculated along with the lithium identity swap, the difference in total electrode-charge, $\Delta Q$ should equal to the amount of the charge transferred during the ET so that $\Delta Q=\delta q=+1 e$ for reduction and $\Delta Q=\delta q=-1 e$ for oxidation, where $Q=\Sigma_{i} A_{i}(t)$ with the index $i$ running for all atoms of both electrodes.

Finally, the correction term $\left(\delta E^{\text {elec }}\right)$ is calculated, considering electric work to transfer all the charges to the anode instead of both electrodes: $\delta E^{\text {elec }}=\left(\delta q^{-}+\delta q^{+}\right) \Psi^{-}-\left(\delta q^{-} \Psi^{-}+\right.$ $\left.\delta q^{+} \Psi^{+}\right)$with the constraint of total transferred charge conservation $\left(\delta q^{-}+\delta q^{+}=\delta q\right)$. Here, $\delta q^{-}$is the charge transferred to the anode at $\Psi^{-}$, and $\delta q^{+}$is the charge transferred to the cathode at $\Psi^{+}$. With no bias potential $\left(\Psi^{-}=\Psi^{+}\right), \delta E^{\text {elec }}=0$. In a slit-like geometry, there is the well-known linear relation between the amount of charges transferred to each of the electrodes and the location of $\mathrm{Li}$ redox-species in a simulation cell. ${ }^{56,57}$ For $\mathrm{Li}^{+}$reduction, such linear relations should be $\delta q^{-}=-e\left(L_{z}^{-1} z-0.5\right)$ and $\delta q^{+}=e\left(L_{z}^{-1} z+0.5\right)$, where $z$ is the $z$ position of the $\mathrm{Li}^{+}$ion, and $L_{z}$ is the length of the simulation box along $z$ axis, determined by a distance between atoms in the electrolyte-exposed layer of each electrode. Then, $\delta E^{\text {elec }}=-e\left(L_{z}^{-1} z+0.5\right) \Delta \Psi$, where $\Delta \Psi$ is a bias potential. To obtain such linear relations for the transferred charges, the vacuum region of double size to the simulation cell along $z$ direction is required when the electrode-charge polarization is recalculated.

\subsection{Free energy curves for an electrochemical ET}

The free energy curves, $F(\Delta E)$ associated with an electrochemical ET are calculated using the following equations in Ref 56:

$$
\begin{aligned}
& F_{o}(\Delta E)=-k_{B} T \ln P_{o}(\Delta E)+\bar{F}_{o}, \\
& F_{r}(\Delta E)=-k_{B} T \ln P_{r}(\Delta E)+\bar{F}_{r},
\end{aligned}
$$


where subindices, $o$ and $r$, stand for $\mathrm{Li}^{+}$and $\mathrm{Li}^{0}$, respectively. The minimum of $F_{o}(\Delta E)$ is set to be zero $\left(\bar{F}_{o}=0\right)$, so the relative vertical shift of the free energy curves is determined by $\bar{F}_{r}$. Both reorganization energy $(\lambda)$ and thermodynamic driving force $(\Delta F)$ are calculated using the linear-response assumptions:

$$
\lambda=\frac{\langle\Delta E\rangle_{r}-\langle\Delta E\rangle_{o}}{2} \quad \Delta F=\frac{\langle\Delta E\rangle_{r}+\langle\Delta E\rangle_{o}}{2} .
$$

Then, the curvature of the parabolic free energies is $1 / 2 \lambda$ regardless of redox species according to the linear response theory. In order to overcome the limited sampling window in our equilibrium simulations, non-equilibrium points are added according to Zwanzig relation: ${ }^{56,58}$

$$
F_{\mathrm{o}}(\Delta E)-F_{\mathrm{r}}(\Delta E)=\Delta E
$$

which linearly relates two free energies to each other.

\subsection{Normalized local density calculations}

The normalized local density, $\rho_{n}(d)$, of atoms as a function of distance from the anode, $d$, is calculated for an atom of $i$-species electrolyte:

$$
\rho_{n}(d)=\frac{\rho_{i}(d)}{\rho_{i}}=\frac{\left\langle\sum_{j=1}^{N_{i}} \delta\left(d-z_{j}^{i}+z_{w}\right)\right\rangle / L_{x} L_{y}}{N_{i} / L_{x} L_{y} L_{z}}
$$

where $\langle\cdots\rangle$ represents the ensemble average, $z_{j}^{i}$ is $z$ position of $j^{\text {th }}$ atom of $i$-species $(j=$ $\left.1,2, \cdots, N_{i}\right), N_{i}$ is the total number of atoms of $i$-species electrolyte, $z_{w}$ is $z$ position of atoms in an electrolyte-exposed layer of the anode, and $L_{k}$ is the length of the simulation box along each direction, $k$ for $k \in\{x, y, z\} . L_{z}$ is determined by a distance between atoms in the electrolyte-exposed layer of each electrode. 


\section{Results and Discussion}

Recent experimental studies have reported that polyethers including PEO and P(2EO-MO) are found to be effective in electrode passivation, enhancing cycling stability, which results from in-situ polymerization via ring-opening reactions of 1,2-dioxolane (DOL). ${ }^{19-22}$ In-situ polymerization enables to overcome poor contact issue that happens when the polymers are prepared ex-situ. Despite their similar chemical composition, they exhibit different lithiumion solvation motifs which influence the resulting ion transport. ${ }^{59}$

\subsection{Lithium-ion solvation at bulk}
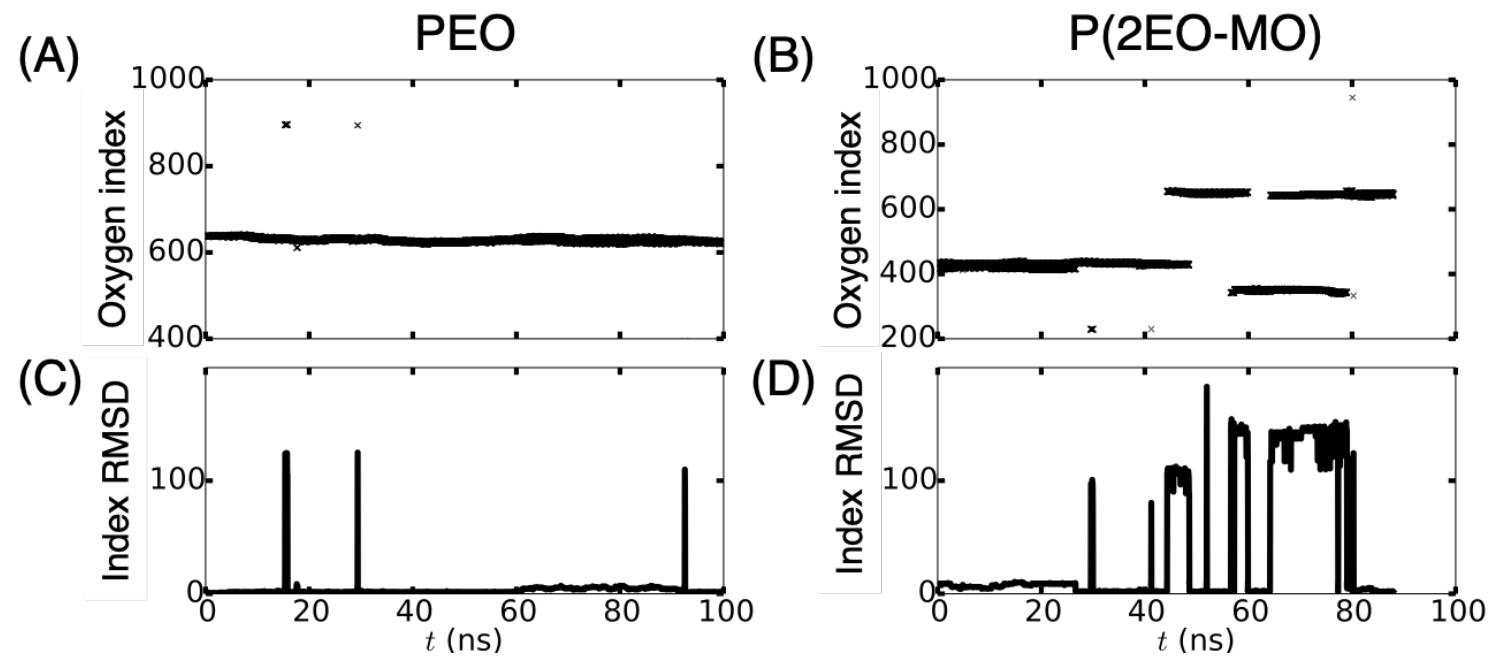

Figure 3: Lithium-ion coordination environment at infinite dilution. Index of oxygens of (A) bulk PEO and (B) bulk $\mathrm{P}(2 \mathrm{EO}-\mathrm{MO})$ that forms the first solvation shell of a representative lithium-ion. Root-mean-square-deviation of the indices (i.e., index RMSD) of the oxygens of (C) PEO and (D) P(2EO-MO). PEO exhibits primarily intra-segmental solvation of the lithium ion, whereas $\mathrm{P}(2 \mathrm{EO}-\mathrm{MO})$ exhibits greater inter-segmental character.

We begin by considering the lithium-ion solvation in the $\mathrm{PEO}$ and $\mathrm{P}(2 \mathrm{EO}-\mathrm{MO})$ bulk polymers. Fig. 3 reveals markedly different lithium-ion solvation structures for the bulk polymers. Ether oxygen atoms of $\mathrm{PEO}$ and $\mathrm{P}(2 \mathrm{EO}-\mathrm{MO})$ are traced as a function of time, $t$, which forms the first solvation shell of a lithium-ion whose distance from the lithium-ion is less than $2.7 \AA$ (the first minimum of radial distribution function between them). The 
oxygen atoms are numbered sequentially from the beginning of each polymer strand. As was shown previously, ${ }^{44,60}$ Fig. 3A exhibits a strap-like structure for PEO that is consistent with lithium-ion solvation by the oxygen atoms along a single continuous segment of the PEO chain. In contrast, Fig. 3B indicates that in bulk $\mathrm{P}(2 \mathrm{EO}-\mathrm{MO})$, the lithium-ions are solvated by two distant segments along the backbone of the polymer, consistent with a two-chain motif for lithium-ion solvation that has previously been noted for other polyesters. ${ }^{44,60}$ As opposed to the one-chain motif of $\mathrm{PEO}$, the reason for the two-chain motif of $\mathrm{P}(2 \mathrm{EO}-\mathrm{MO})$ is a single methylene unit that separates two oxygen atoms, which is not consistent with lithium-ion solvation. ${ }^{59}$ Fig. 3C and D display root-mean-square-deviation (RMSD) of a set of indices for the oxygen that solvate a lithium-ion as a function of time, $t$, which discriminates between the lithium-ion solvation motifs of a polymer. For an $i^{\text {th }}$ lithium-ion,

$$
\mathrm{RMSD}=\sqrt{\frac{1}{N_{o}} \sum_{j \in \mathbb{S}_{i}}\left(O_{j}-\frac{1}{N_{o}} \sum_{j \in \mathbb{S}_{i}} O_{j}\right)^{2}}
$$

where $O_{j}$ is an index of $j^{\text {th }}$ oxygen that forms the first solvation shell of the $i^{\text {th }}$ lithium-ion, $\mathbb{S}_{i}$ is a set of the oxygen atoms whose number of elements is $N_{O}$. The lithium-ion solvation is defined as a one-chain motif if RMSD is less than 5, or a two-chain motif otherwise. For instance, RMSD $=2$ if 7 consecutive oxygen atoms of PEO solvate a lithium-ion. Table 1 displays the fraction of each lithium-ion solvation motif, quantified via RMSD. About $90 \%$ of lithium ions are solvated by a single segment in $\mathrm{PEO}$, as opposed to $63 \%$ in $\mathrm{P}(2 \mathrm{EO}-\mathrm{MO})$ which exhibits both intra- and inter-segmental solvation motifs.

Table 1: Fraction of lithium-ion solvation motifs in bulk electrolytes at finite concentration

\begin{tabular}{|c||c|c|c|c|}
\hline & PEO & P(2EO-MO) & G4 & DME $^{a}$ \\
\hline one-chain & $0.90(1)$ & $0.63(2)$ & $0.33(1)$ & $0.03(1)$ \\
two-chain & $0.10(1)$ & $0.37(2)$ & $0.67(1)$ & $0.60(4)$ \\
\hline
\end{tabular}

${ }^{a}$ The remaining fraction is associated with multi-chain solvation more than two.

Statistical errors of the final digit are indicated in parentheses. 


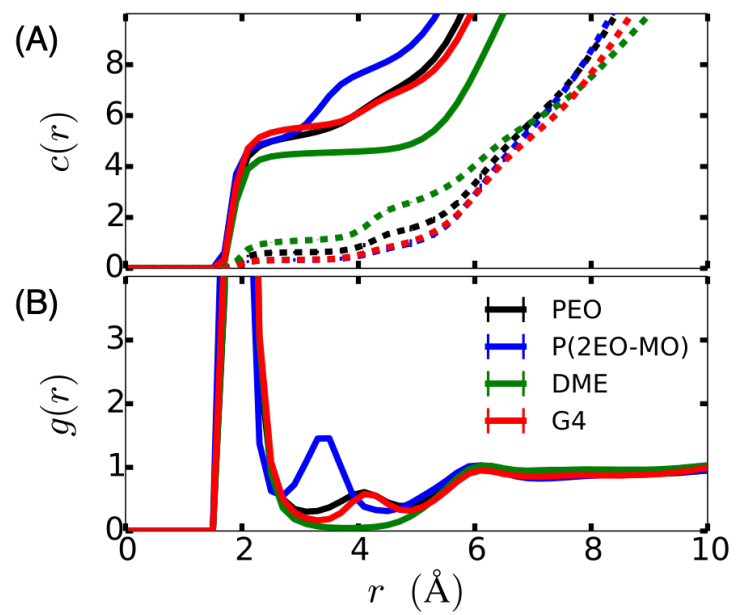

Figure 4: Cumulative (A) and radial (B) $\mathrm{Li}^{+}$-oxygen distribution functions for the bulk electrolytes. Solid lines represent oxygen atoms of the electrolyte, and dotted lines represent oxygen atoms of $\mathrm{TFSI}^{-}$anion in each electrolyte.

Additional insight into these lithium-ion solvation structures is provided in Fig. 4 that most of the lithium-ions in bulk polymers are free from ion-pairing, and solvated solely by the polymer electrolytes. The cumulative number, $c(r)$ of neighbor oxygen atoms around a lithium-ion is calculated as a function of the interatomic distance, $r$ between the ion and the oxygen atom. The first solvation shell of the lithium-ion includes 5 or 6 ether oxygen atoms of PEO that is the only chemical moiety that interacts preferentially with lithium ions. ${ }^{44,60}$ According to $c(r)$, despite the differences in lithium-ion solvation motif and ether oxygen density, both two polymers provide a similar number of ether oxygens in the first solvation shell for a lithium-ion, which outcompetes TFSI oxygen. The second peak of the radial distribution function, $g(r)$ for $\mathrm{P}(2 \mathrm{EO}-\mathrm{MO})$, not present for $\mathrm{PEO}$, supports that not all three ether oxygens in its monomer unit participate in chelating the lithium-ion but one ether oxygen atom is excluded in lithium-ion solvation.

Fig. 4 further shows that the molecular solvents, DME and G4 interact locally with a lithium-ion in a similar way of their associated polymers. The lithium-ion solvation structure is almost the same between PEO and G4. As opposed to the one-chain solvation of PEO, two-third of lithium-ions are solvated by two G4 molecules with the remaining ones by a 
single G4 molecule (Table 1). No single DME molecule by nature can fully solvate a lithiumion, which instead requires at least two DME molecules. The ether oxygen in both DME and G4 outcompetes TFSI oxygen for lithium-ion solvation as it does in both polymers. Taken together, these findings suggest that observed differences between the polymers and their associated molecular solvents are primarily due to the polymer connectivity and resulting solvation motifs.

\subsection{Ion solvation at the anode interface}

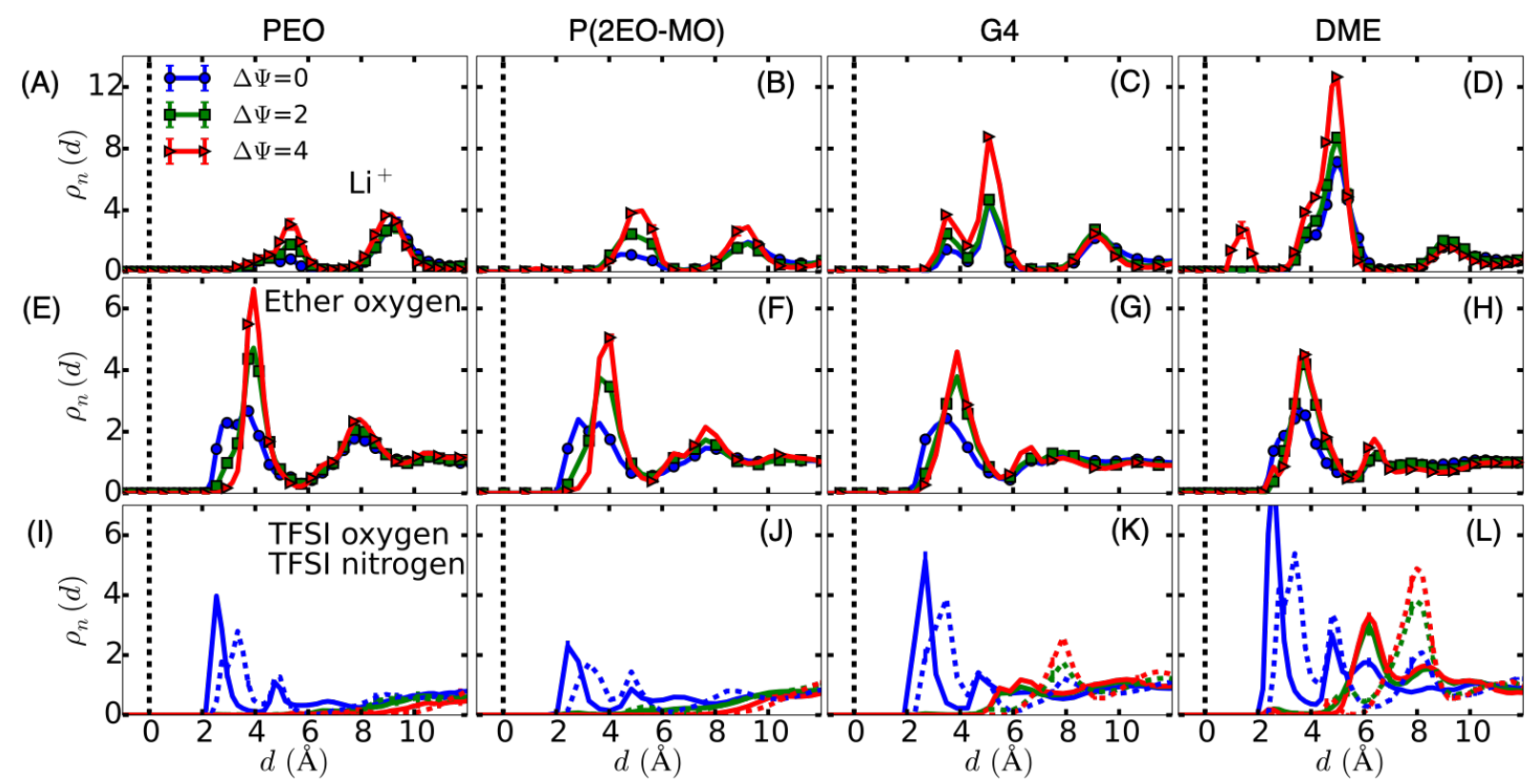

Figure 5: The normalized local density (Eq. 7) of the ions and electrolytes as a function of distance, $d$ from the anode at various bias potentials, $\Delta \Psi$. The columns of panels correspond to the various considered electrolytes. Row (A-D) presents the distribution for the $\mathrm{Li}^{+}$ions; Row (E-H) presents the distribution for ether oxygen of the electrolyte; Row (I-L) presents the distribution for TFSI ${ }^{-}$ions. In panels (I-L), solid lines represent oxygen atoms of TFSI ${ }^{-}$ anion, and dotted lines represent a nitrogen atom of TFSI- anion. In all panels, the black vertical line indicates the location of the electrolyte-exposed layer of the anode.

Fig. 5 plots the normalized density of $\mathrm{Li}^{+}$ion, $\mathrm{TFSI}^{-}$ion, and electrolyte ether oxygens for each of the considered electrolytes. It is seen that with finite bias potentials, $\Delta \Psi>0 \mathrm{~V}$, the EDL forms in all cases, evidenced by the preferential interaction of $\mathrm{Li}^{+}$ions over TFSI ${ }^{-}$ ions with the anode. 
Figure 6 illustrates that the $\mathrm{Li}^{+}$solvation at the interface (i.e., the closest peak to the anode in each case from Fig. 5) is different than in the bulk. As discussed in Table 1, the bulk solvation exhibits a range of one-chain versus two- or higher-chain solvation environments, depending on the electrolyte. For PEO, the interfacial $\mathrm{Li}^{+}$solvation remains dominated by one-chain solvation, and for DME, it remains dominated by two-chain and higher solvation structures. However, for $\mathrm{P}(2 \mathrm{EO}-\mathrm{MO})$, the fraction of one-chain solvation decreases, and for G4, the fraction of one-chain solvation significantly increases in the vicinity of the anode. The results in Fig. 6 are presented for the case of zero bias potential, although the same conclusions hold at non-zero values (Fig. S2 in SI).

Several additional features in $\mathrm{Li}^{+}$ion solvation are worth noting in Fig. 5. Firstly, efficient solvation via the electrolytes leads to a layer of solvent separation between the anode and the $\mathrm{Li}^{+}$, such that the closest peak to the anode is typically at $d>2 \AA$. However, for DME at the highest bias potential, this solvent-separation layer partially breaks down, allowing for direct contact between the $\mathrm{Li}^{+}$ions and the anode, suggesting a greater propensity to form an inner-Helmholtz layer in this electrolyte. This effect is consistent with the fact that DME only allows for two-chain (or multiple-chain) solvation of the $\mathrm{Li}^{+}$ions (Table 1 ), thereby providing less efficient solvation in the confined environment at the interface. Also, we note that the interfacial $\mathrm{Li}^{+}$solvation peak for G4 is split into two sub-peaks, with the closest one dominated by one-chain solvation and with the more distant one dominated by two-chain solvation (Fig. S2 in SI).

Finally, with regard to the TFSI ${ }^{-}$anion solvation, Fig. 5 shows a notable difference between the polymers and molecular liquids. At zero bias, all of the electrolytes show significant peaks near the anode associated with formation of the EDL, and at finite bias potential, these peaks are shifted outward in all cases. For the polymer electrolytes, the anode peak largely flattens to unstructured, bulk-like behavior, whereas for the molecular electrolytes, the anion solvation peak remains sharp and highly structured. Such pronounced differences in the anion solvation at finite potentials may suggest that the polymer electrolytes provide 
an additional barrier for physical access of anions to the anode interface, thereby retarding the detrimental breakdown of anions. ${ }^{61-65}$

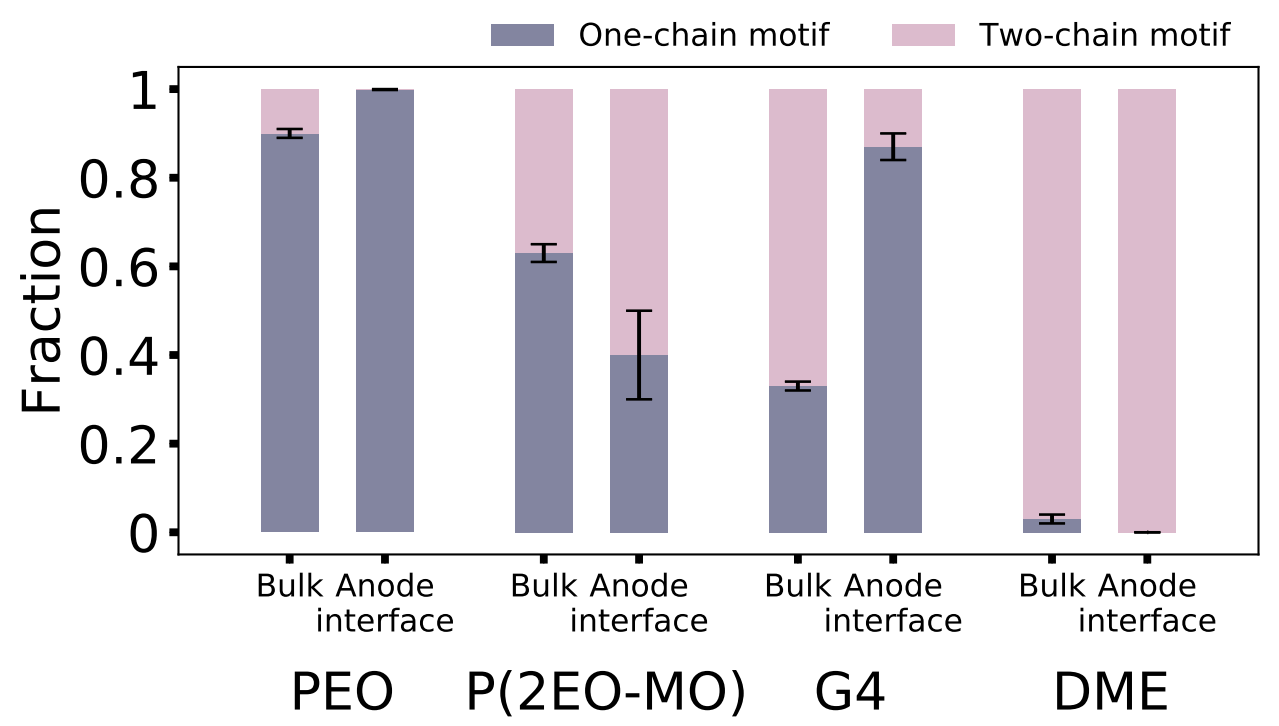

Figure 6: Fraction of $\mathrm{Li}^{+}$ion solvation motifs, in bulk and at the anode interface with zero bias.

\subsection{Electrode-charge polarization and solvent fluctuations}

Just as the presence of electrode influence the solvation structure of the interfacial ions, the ions influence the charge polarization of the electrode. In fact, electrode charge polarization is strongly sensitive to rare fluctuations in the solvent structure. ${ }^{42,53}$

Fig. 7 displays the distribution of induced charge per atom of the anode, where $P(q)=$ $\left\langle\delta\left(q-A_{j}(t)\right)\right\rangle$ with the index $j$ running for all atoms of the anode. For small and zero biasing potentials, the distributions are strongly Gaussian, indicating weak correlations in the interfacial solvent fluctuations. However, at the highest bias potential, all four electrolytes exhibit a non-Gaussian tail at negative charges associates with unexpectedly large chargepolarization fluctuations. This tail is largest for DME, which as seen in Fig. 5 to support a significant population of $\mathrm{Li}^{+}$ions in direct contact with the anode interface. It is striking, however, that the effect also appears in the other electrolytes for which such direct lithium- 

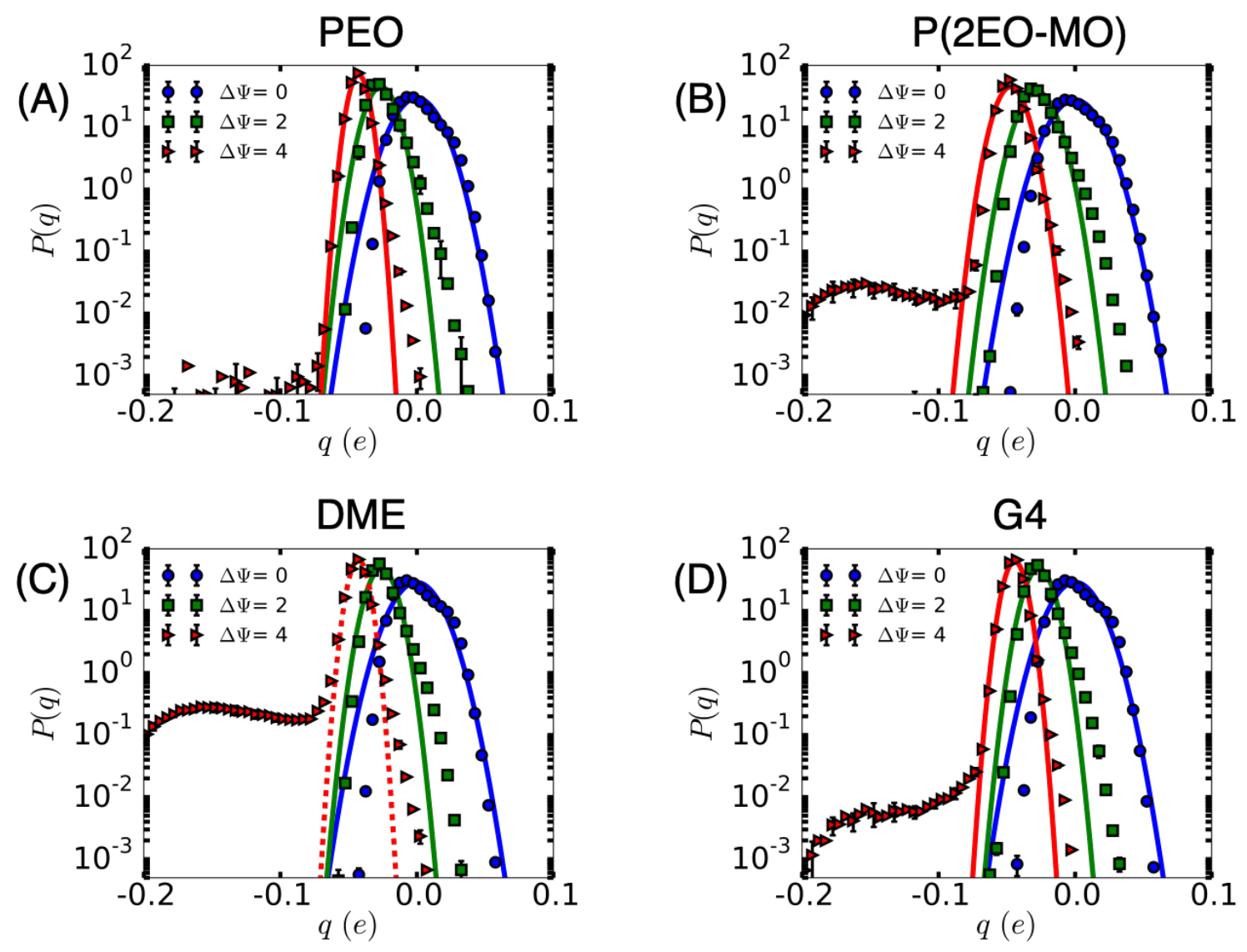

(E)

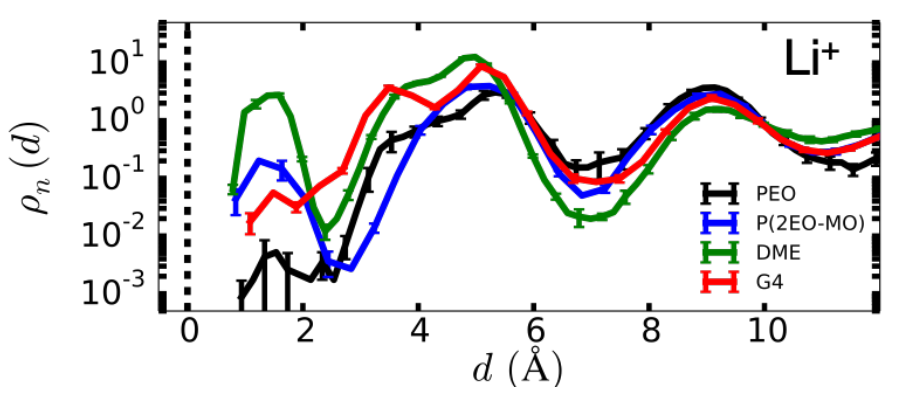

Figure 7: Distribution of induced charge per atom of the anode, for the various electrolytes and bias potentials. (A) PEO. (B) P(2EO-MO). (C) DME. (D) G4. Solid lines represent Gaussian fits. (E) Normalized local density of $\mathrm{Li}^{+}$ions on a log-linear scale at $\Delta \Psi=4 \mathrm{~V}$. 
anode contact were not seen in Fig. 5. Nonetheless, by investigating the ion-distribution plots from Fig. 5 on a log scale (Fig. 7E), it is seen that rare ion-solvation fluctuations do indeed occur for the PEO, P(2EO-MO), and G4 electrolytes, which give rise to this strongly non-Gaussian interfacial behavior. As expected, PEO, which exhibits efficient single-chain solvation of the $\mathrm{Li}^{+}$ions, best preserves solvent separation between $\mathrm{Li}^{+}$and the electrode and exhibits the smallest degree of non-Gaussian behavior in Fig. 7.

\subsection{Outer-sphere ET at the anode interface}

Beyond anion-mediated decomposition, the solvation structure for ions at the electrode interface have significant implications for the redox chemistry and reaction rates that will occur. In the current section, we consider this point from the perspective of the reduction $\mathrm{Li}^{+}$ions via interfacial electron transfer.

In this section, we confine our attention to electrolytes and solvation structures for which the $\mathrm{Li}+$ is solvent-separated from the anode. Specifically, we consider outer-sphere ET for $\mathrm{Li}^{+}$ions at solvent-separated distance of $2 \AA<d<6 \AA$ from the metal anode surface, for which non-adiabatic ET kinetics is applicable. Since the ET rate decays exponentially with the distance from the interface, these $\mathrm{Li}^{+}$ions are expected to be electrochemically reduced at the fastest rate, except for inner-sphere $\mathrm{Li}^{+}$ions that adsorb directly on the anode that requires adiabatic ET kinetics.

Fig. 8 confirms that outer-sphere interfacial electrochemical ET in PEO is well described using the assumption of Gaussian fluctuations for local solvent electric field, as is consistent with the observations in Fig. 7. The left column in Fig. 8 shows that the energy-gap distribution $P(\Delta E)$ for both $\mathrm{Li}^{+}$and $\mathrm{Li}^{0}$ at the $\mathrm{PEO} /$ anode interface are Gaussian in nature. This conclusion is independent of the strength of the bias potential. The right column in Fig. 8 further illustrates this point, with the free-energy surfaces in the energy-gap coordinate exhibiting parabolic form; in panels D-F, the solid lines correspond to the assumption of Gaussian fluctuations (Eq. 5) and are in excellent agreement with the simulation datapoints. 
PEO

(A)

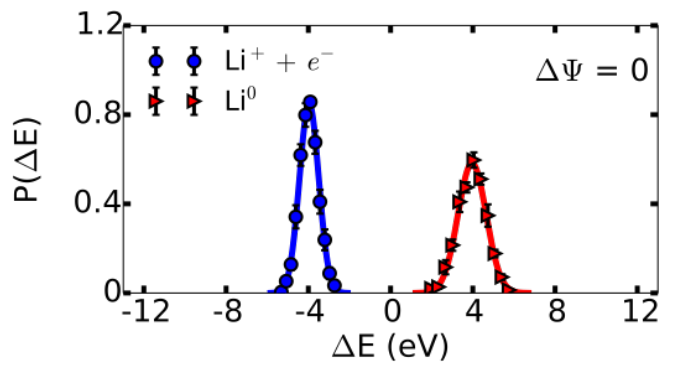

(B)

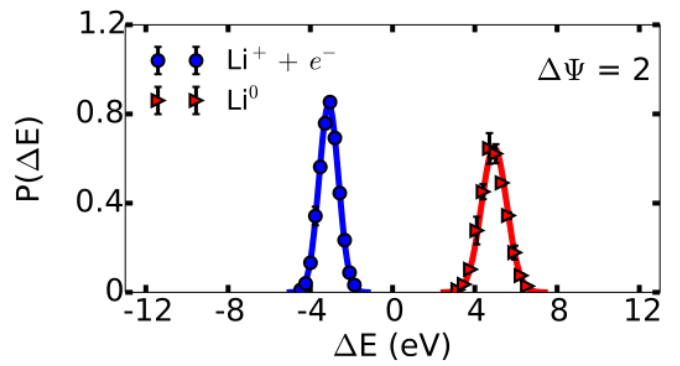

(C)

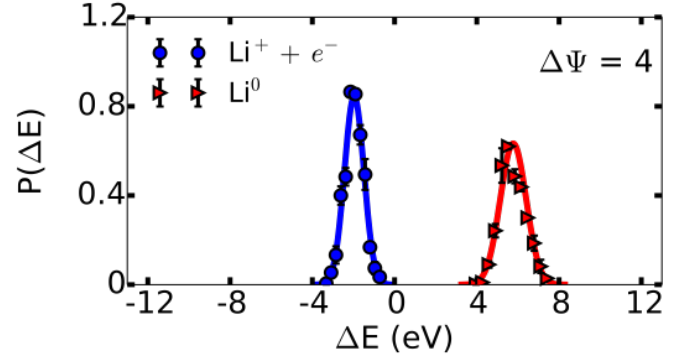

(D)

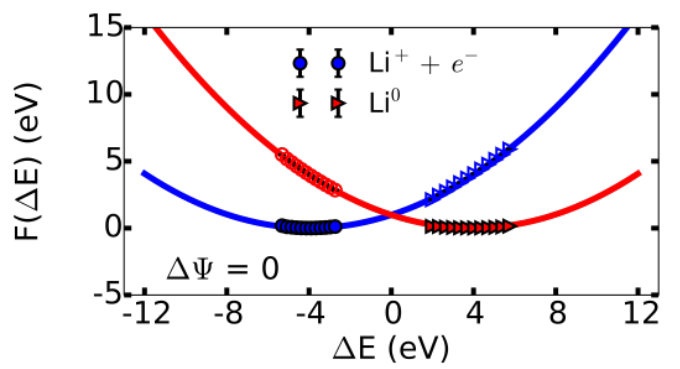

(E)

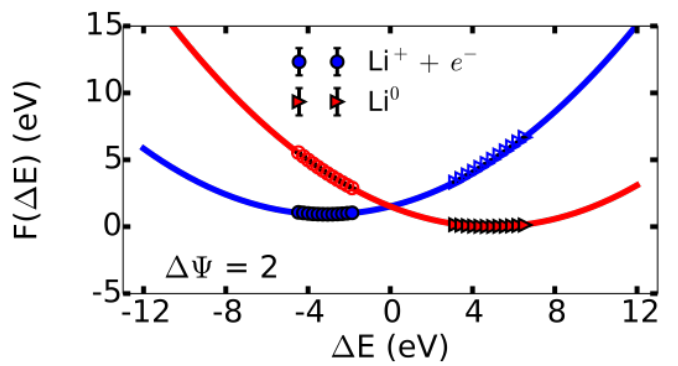

(F)

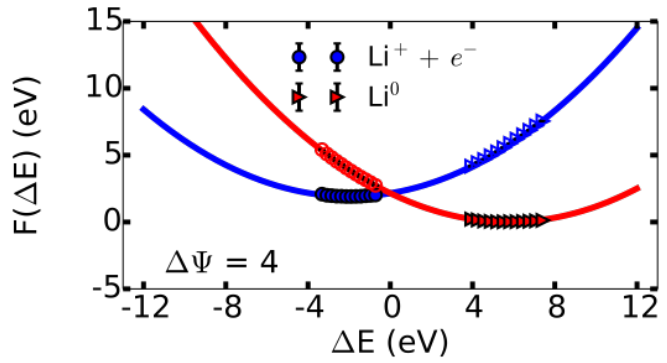

Figure 8: Lithium electroreduction in PEO at the anode interface. (A, B, and C) Probability distributions $P(\Delta E)$ of the vertical energy gap $\Delta E$, and $(\mathrm{D}, \mathrm{E}$, and $\mathrm{F})$ their associated free energy curves for both the $\mathrm{Li}^{+}$and $\mathrm{Li}^{0}$ in PEO. Results are shown for various bias potentials. Solid lines in (D, E, and F) are parabolic curves using linear-response assumptions in Eq. 5. 
These observations validate the use of linear-response theory for the description of the outersphere interfacial ET in these systems. Similar results are found for the outer-sphere ET reactions in the other electrolytes considered.

\section{Conclusions}

Interfacial stability has been one of major bottlenecks in development of next-generation rechargeable batteries. ${ }^{1-9}$ Functional SEIs passivate an electrode by regulating lithium electrodeposition and by preventing detrimental decomposition reactions of electrolytes. Here, we perform atomic-resolution simulations with constant-potential polarizable electrodes to characterize interfacial electron-transfer kinetics, including lithium-ion solvation structures and solvent reorganization effects as a function of applied electrode potential. Linear ether homopolymers are studied as a chemically and structurally well-defined proxy for polymeric SEIs, including PEO and P(2EO-MO), along with the ethereal molecular solvents G4 and DME.

Simulations show that all considered electrolytes except for DME provide a solventseparation layer for $\mathrm{Li}^{+}$ions at the anode interface, with both one-chain and two-chain solvation environments. Efficient passivation may facilitate homogeneous lithium electroreduction. ${ }^{6,7}$ Rare fluctuations in solvent structure strongly affect electrode-charge polarization such that direct lithium-anode contact gives rise to a tail of electrode-charge polarization. However, at the highest bias potential, DME allows for direct contact between the $\mathrm{Li}^{+}$ions and the anode with greater propensity. Further, the substantial difference between the polymers and molecular solvents is found in solvation of the interfacial TFSI ${ }^{-}$anions; the polymer electrolytes largely expel $\mathrm{TFSI}^{-}$anions from the anode interface, which could delay further

detrimental anion breakdown. ${ }^{2}$ Finally, the assumptions of Gaussian solvent fluctuations for outer-sphere ET for $\mathrm{Li}^{+}$ions are found to be robust in these systems, although both transient and metastable direct-contact pairs between the anode and the $\mathrm{Li}^{+}$ions may be expected 
to dominate the ET kinetics through an inner-sphere mechanism. The robustness of these observations across the range of polymer and molecule electrolytes here may generalize to other interfacial environments. ${ }^{18,37,66}$

\section{Acknowledgement}

TFM acknowledges support from the Center for Synthetic Control Across Length-Scales for Advancing Rechargeables (SCALAR), an Energy Frontier Research Center funded by the U.S. Department of Energy, Office of Science, Basic Energy Sciences under Award No. DE-SC0019381. Computational resources were provided by the National Energy Research Scientific Computing Center (NERSC), a DOE Office of Science User Facility supported by the DOE Office of Science under contract DE- AC02-05CH11231. 


\section{References}

(1) Tarascon, J. M.; Armand, M. Nature 2001, 414, 359-367.

(2) Xu, K. Chem. Rev. 2014, 114, 11503-11618.

(3) Nitta, N.; Wu, F.; Lee, J. T.; Yushin, G. Materials Today 2015, 18, 252-264.

(4) Gauthier, M.; Carney, T. J.; Grimaud, A.; Giordano, L.; Pour, N.; Chang, H.-H.; Fenning, D. P.; Lux, S. F.; Paschos, O.; Bauer, C.; Maglia, F.; Lupart, S.; Lamp, P.; Shao-Horn, Y. The Journal of Physical Chemistry Letters 2015, 6, 4653-4672.

(5) Varzi, A.; Raccichini, R.; Passerini, S.; Scrosati, B. J. Mater. Chem. A 2016, 4, 1725117259.

(6) Tikekar, M. D.; Choudhury, S.; Tu, Z.; Archer, L. A. Nature Energy 2016, 1, 1-7.

(7) Lin, D.; Liu, Y.; Cui, Y. Nature Nanotechnology 2017, 12, 194-206.

(8) Gauthier, M.; Carney, T. J.; Grimaud, A.; Giordano, L.; Pour, N.; Chang, H.-H.; Fenning, D. P.; Lux, S. F.; Paschos, O.; Bauer, C.; Maglia, F.; Lupart, S.; Lamp, P.; Shao-Horn, Y. The Journal of Physical Chemistry Letters 2015, 6, 4653-4672.

(9) Giordano, L.; Karayaylali, P.; Yu, Y.; Katayama, Y.; Maglia, F.; Lux, S.; Shao-Horn, Y. The Journal of Physical Chemistry Letters 2017, 8, 3881-3887.

(10) Pinson, M. B.; Bazant, M. Z. Journal of The Electrochemical Society 2012, 160, A243A250.

(11) Peled, E.; Menkin, S. Journal of The Electrochemical Society 2017, 164, A1703-A1719.

(12) Cheng, X.-B.; Zhang, R.; Zhao, C.-Z.; Wei, F.; Zhang, J.-G.; Zhang, Q. Adv. Sci. 2016, 3, 1500213 . 
(13) An, S. J.; Li, J.; Daniel, C.; Mohanty, D.; Nagpure, S.; Wood, D. L. Carbon 2016, 105, $52-76$.

(14) Ramos-Sanchez, G.; Soto, F. A.; de la Hoz, J. M. M.; Liu, Z.; Mukherjee, P. P.; ElMellouhi, F.; Seminario, J. M.; Balbuena, P. B. Journal of Electrochemical Energy Conversion and Storage 2016, 13, 031002.

(15) Verma, P.; Maire, P.; Novák, P. Electrochimica Acta 2010, 55, 6332-6341.

(16) Bülter, H.; Peters, F.; Schwenzel, J.; Wittstock, G. Angewandte Chemie (International ed. in English) 2014, 53, 10531-10535.

(17) Zachman, M. J.; Tu, Z.; Choudhury, S.; Archer, L. A.; Kourkoutis, L. F. Nature 2018, 560,345 .

(18) Li, Y.; Qi, Y. Energy \& Environmental Science 2019, 12, 1286-1295.

(19) Aurbach, D.; Youngman, O.; Dan, P. Electrochimica Acta 1990, 35, 639-655.

(20) La Monaca, A.; De Giorgio, F.; Soavi, F.; Tarquini, G.; Di Carli, M.; Paolo Prosini, P.; Arbizzani, C. ChemElectroChem 2018, 5, 1272-1278.

(21) Zhao, Q.; Liu, X.; Stalin, S.; Khan, K.; Archer A., L. Nature energy 2019,

(22) Choudhury, S.; Tu, Z.; Nijamudheen, A.; Zachman, M. J.; Stalin, S.; Deng, Y.; Zhao, Q.; Vu, D.; Kourkoutis, L. F.; Mendoza-Cortes, J. L.; Archer, L. A. Nature communications 2019, 10, 3091.

(23) Stark, J. K.; Ding, Y.; Kohl, P. A. Journal of The Electrochemical Society 2011, 158, A1100-6.

(24) Zhao, H.; Zhou, X.; Park, S.-J.; Shi, F.; Fu, Y.; Ling, M.; Yuca, N.; Battaglia, V.; Liu, G. Journal of Power Sources 2014, 263, 288-295. 
(25) Zheng, J.; Engelhard, M. H.; Mei, D.; Jiao, S.; Polzin, B. J.; Zhang, J.-G.; Xu, W. Nature Energy 2017, 2, 17012-8.

(26) Li, Y.; Li, Y.; Pei, A.; Yan, K.; Sun, Y.; Wu, C.-L.; Joubert, L.-M.; Chin, R.; Koh, A. L.; Yu, Y.; Perrino, J.; Butz, B.; Chu, S.; Cui, Y. Science 2017, 358, 506-510.

(27) An, Y.; Zhang, Z.; Fei, H.; Xu, X.; Xiong, S.; Feng, J.; Ci, L. Journal of Power Sources 2017, 363, 193-198.

(28) Rubinstein, M.; Colby, R. Polymer Physics; OUP Oxford, 2003.

(29) Ren, C.-L.; Nakamura, I.; Wang, Z.-G. Macromolecules 2015, 49, 425-431.

(30) Irwin, M. T.; Hickey, R. J.; Xie, S.; Bates, F. S.; Lodge, T. P. Macromolecules 2016, 49, 4839-4849.

(31) Ferguson, T. R.; Bazant, M. Z. Journal of The Electrochemical Society 2012, 159, A1967-A1985.

(32) Matyushov, D. V. Acc. Chem. Res. 2007, 40, 294-301.

(33) Vuilleumier, R.; Tay, K. A.; Jeanmairet, G.; Borgis, D.; Boutin, A. J. Am. Chem. Soc. 2011, 134, 2067-2074.

(34) Laborda, E.; Henstridge, M. C.; Batchelor-McAuley, C.; Compton, R. G. Chem. Soc. Rev. 2013, 42, 4894-4950.

(35) Petersen, M. K.; Kumar, R.; White, H. S.; Voth, G. A. The Journal of Physical Chemistry C 2012, 116, 4903-4912.

(36) Borodin, O.; Olguin, M.; Ganesh, P.; Kent, P. R. C.; Allen, J. L.; Henderson, W. A. Physical Chemistry Chemical Physics 2016, 18, 164-175.

(37) Lopez, J.; Pei, A.; Oh, J. Y.; Wang, G.-J. N.; Cui, Y.; Bao, Z. Journal of the American Chemical Society 2018, 140, 11735-11744. 
(38) Limmer, D. T.; Willard, A. P.; Madden, P.; Chandler, D. Proceedings of the National Academy of Sciences 2013, 110, 4200-4205.

(39) Willard, A. P.; Reed, S. K.; Madden, P. A.; Chandler, D. Faraday Discussions 2009, $141,423-441$.

(40) Limmer, D. T.; Willard, A. P. Chem. Phys. Lett. 2015, 620, 144-150.

(41) Limmer, D. T.; Willard, A. P.; Madden, P. A.; Chandler, D. The Journal of Physical Chemistry C 2015, 119, 24016-24024.

(42) Kattirtzi, J. A.; Limmer, D. T.; Willard, A. P. Proceedings of the National Academy of Sciences of the United States of America 2017, 114, 13374-13379.

(43) Plimpton, S. Journal of Computational Physics 1995, 117, 1 - 19.

(44) Webb, M. A.; Savoie, B. M.; Wang, Z.-G.; Miller III, T. F. Macromolecules 2015, 48, $7346-7358$.

(45) Stubbs, J. M.; Potoff, J. J.; Siepmann, J. I. The Journal of Physical Chemistry B 2004, 108, 17596-17605.

(46) Xu, M.; Chen, J.; Zhang, C.; Du, Z.; Mi, J. Phys. Chem. Chem. Phys. 2011, 13, 21084-21092.

(47) Lopes, J. N. C.; Pádua, A. A. H. The Journal of Physical Chemistry B 2004, 108, $16893-16898$.

(48) Siepmann, J. I.; Sprik, M. The Journal of Chemical Physics 1995, 102, 511-524.

(49) Reed, S. K.; Lanning, O. J.; Madden, P. A. The Journal of Chemical Physics 2007, 126, 084704-14.

(50) Gingrich, T. R.; Wilson, M. Chemical Physics Letters 2010, 500, 178-183. 
(51) Wang, Z.; Yang, Y.; Olmsted, D. L.; Asta, M.; Laird, B. B. The Journal of Chemical Physics 2014, 141, 184102.

(52) Heinz, H.; Vaia, R. A.; Farmer, B. L.; Naik, R. R. The Journal of Physical Chemistry C 2008, 112, 17281-17290.

(53) Shayeghi, A.; Krähling, S.; Hörtz, P.; Johnston, R. L.; Heard, C. J.; Schäfer, R. The Journal of Physical Chemistry C 2017, 121, 21354-21363.

(54) Yeh, I.-C.; Berkowitz, M. L. The Journal of Chemical Physics 1999, 111, 3155-3162.

(55) Hwang, J.-K.; Warshel, A. J. Am. Chem. Soc. 1987, 109, 715-720.

(56) Reed, S. K.; Madden, P. A.; Papadopoulos, A. The Journal of Chemical Physics 2008, 128, 124701.

(57) Zahn, M. Am. J. Phys. 1976, 44, 1132-1134.

(58) Blumberger, J.; Sprik, M. Lect. Notes Phys. 2006, 704, 481-506.

(59) Zheng, Q.; Pesko, D. M.; Savoie, B. M.; Timachova, K.; Hasan, A. L.; Smith, M. C.; Miller, T. F.; Coates, G. W.; Balsara, N. P. Macromolecules 2018, 51, 2847-2858.

(60) Webb, M. A.; Jung, Y.; Pesko, D. M.; Savoie, B. M.; Yamamoto, U.; Coates, G. W.; Balsara, N. P.; Wang, Z.-G.; Miller, T. F. ACS Central Science 2015, 1, 198-205.

(61) Vatamanu, J.; Borodin, O.; Smith, G. D. The Journal of Physical Chemistry C 2011, $116,1114-1121$.

(62) Suo, L.; Borodin, O.; Gao, T.; Olguin, M.; Ho, J.; Fan, X.; Luo, C.; Wang, C.; Xu, K. Science 2015, 350, 938-943.

(63) Fan, X.; Chen, L.; Ji, X.; Deng, T.; Hou, S.; Chen, J.; Zheng, J.; Wang, F.; Jiang, J.; Xu, K.; Wang, C. Chem 2018, 4, 174-185. 
(64) Wang, M.; Huai, L.; Hu, G.; Yang, S.; Ren, F.; Wang, S.; Zhang, Z.; Chen, Z.; Peng, Z.; Shen, C.; Wang, D. The Journal of Physical Chemistry C 2018, 122, 9825-9834.

(65) Yan, C.; Cheng, X.-B.; Tian, Y.; Chen, X.; Zhang, X.-Q.; Li, W.-J.; Huang, J.-Q.; Zhang, Q. Advanced Materials 2018, 30, 1707629.

(66) Li, B.; Wang, Y.; Yang, S. Advanced Energy Materials 2018, 8, 1702296. 


\section{Graphical TOC Entry}

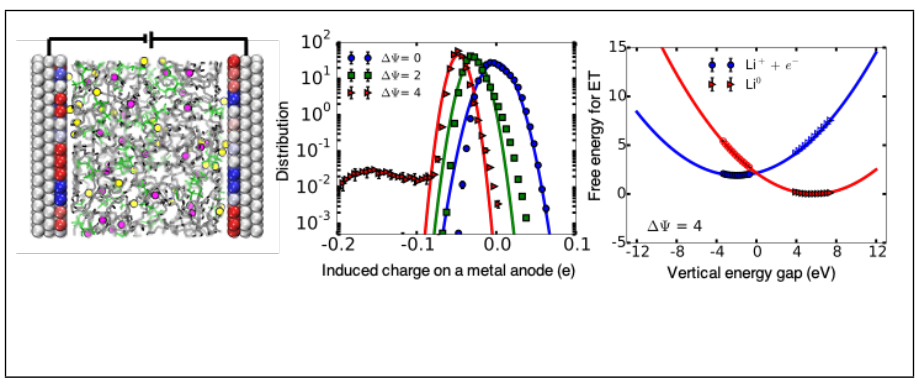




\title{
Supporting Information for
}

\section{Interfacial Electron Transfer and Ion Solvation in the Solid Electrolyte Interphase}

\author{
Jeongmin Kim, ${ }^{\dagger}$ Brett M. Savoie,,$^{\ddagger}$ and Thomas F. Miller $\mathrm{III}^{*, \dagger}$ \\ $\dagger$ Division of Chemistry and Chemical Engineering, California Institute of Technology, \\ Pasadena, California, USA \\ $\ddagger$ School of Chemical Engineering, Purdue University, West Lafayette, Indiana, USA \\ E-mail: tfm@caltech.edu
}




\section{S1 Mean electric potential}

(A)

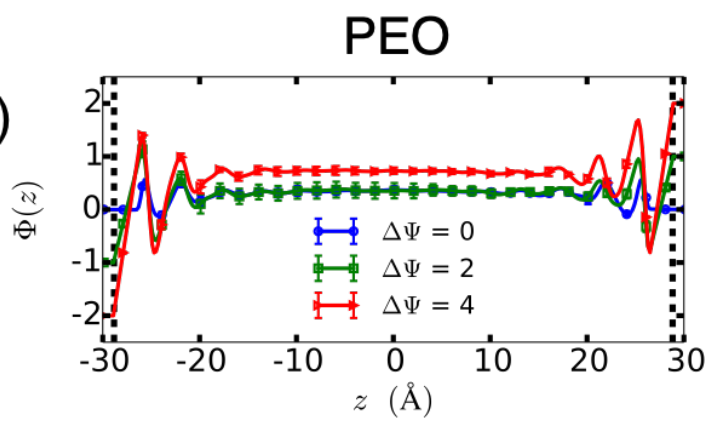

(C)

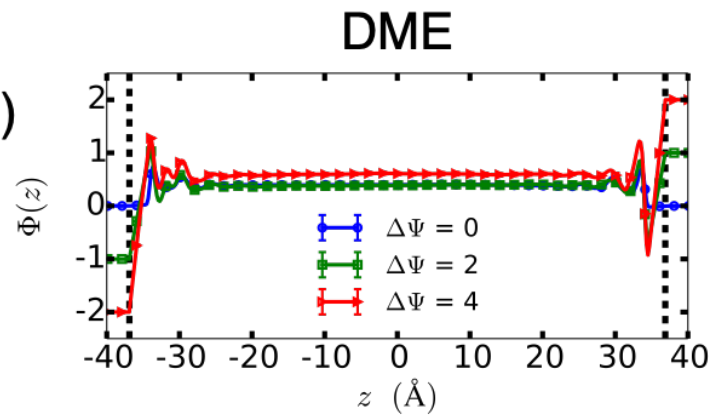

(B)
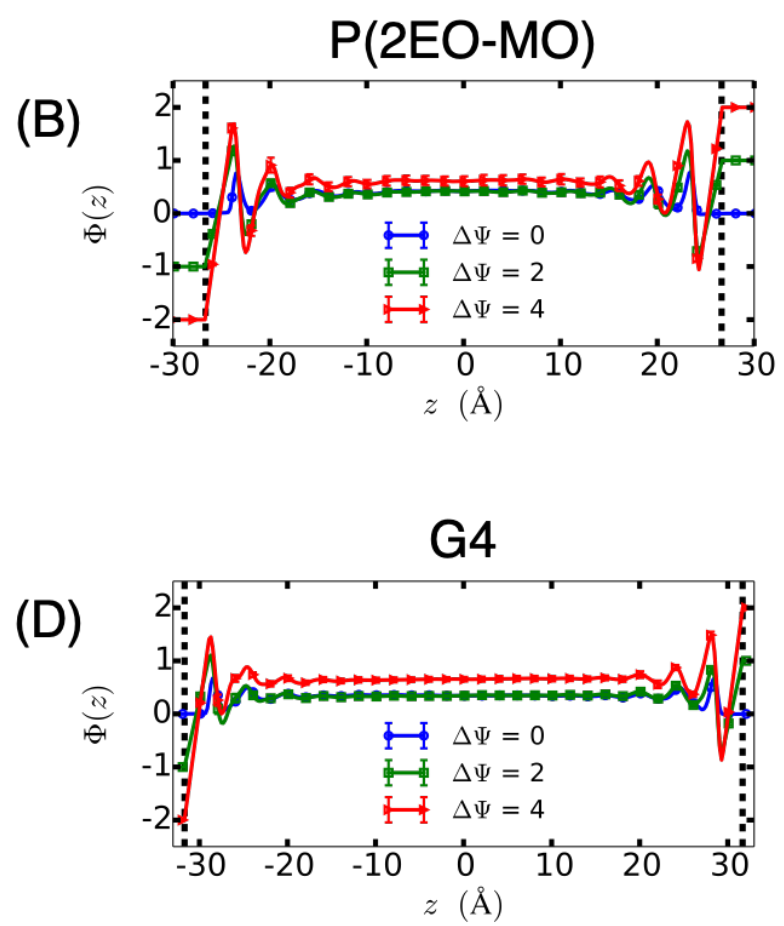

Figure S1: Mean electric potential across the simulation cell for the various electrolytes and bias potentials. In all panels, the black vertical line indicates the location of the electrolyteexposed layer of the anode.

Fig. S1 shows mean electric potential, $\Phi(z)$ calculated, solving Poisson equation numerically across the simulation cell. ${ }^{1,2}$

$$
\frac{d^{2} \Phi(z)}{d z^{2}}=-\frac{\rho(z)}{\epsilon_{0}}
$$

where $\rho(z)$ is the mean charge density averaged over a xy plane, and $\epsilon_{0}$ is vacuum permittivity. In all considered electrolytes, $\Phi(z)$ oscillates at the electrode interface due to the electrical double layer. After the screening from the interfacial ions, $\Phi(z)$ reaches the plateau. 


\section{S2 Spatially-resolved lithium-ion solvation motif at the anode interface}

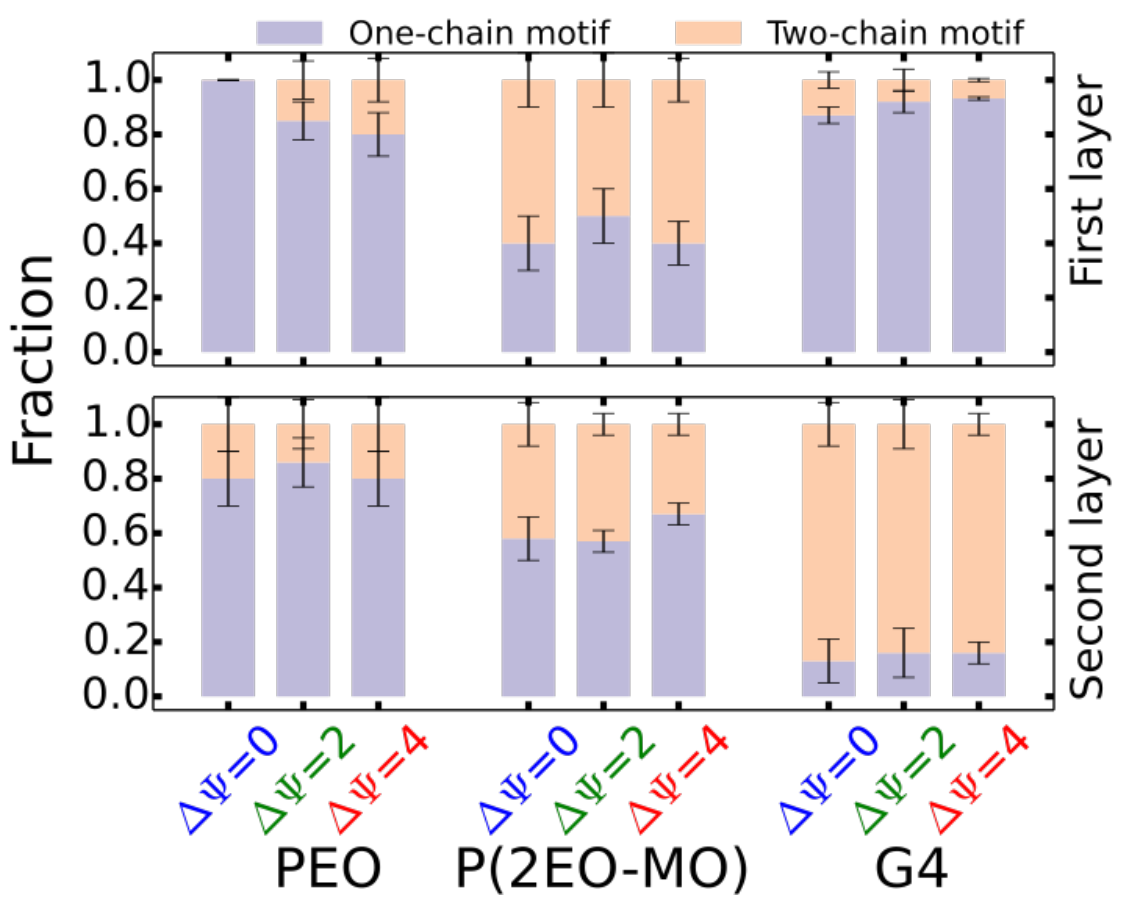

Figure S2: Spatially-resolved lithium-ion solvation motif at the anode interface. A top panel is for the first layer of interfacial lithium-ions, and a bottom panel is for the second layer of interfacial lithium-ions. For $\mathrm{PEO}$ and $\mathrm{P}(2 \mathrm{EO}-\mathrm{MO})$, the first layer is a region of $d \in(2,7)$, and the second layer is a region of $d \in[7,11)$. For $\mathrm{G} 4$, the first layer is a region of $d \in(2,4.5)$, and the second layer is a region of $d \in[4.5,7)$. The distance $(d)$ is from the anode. 


\section{S3 Outer-sphere ET at the anode interface}

(A)

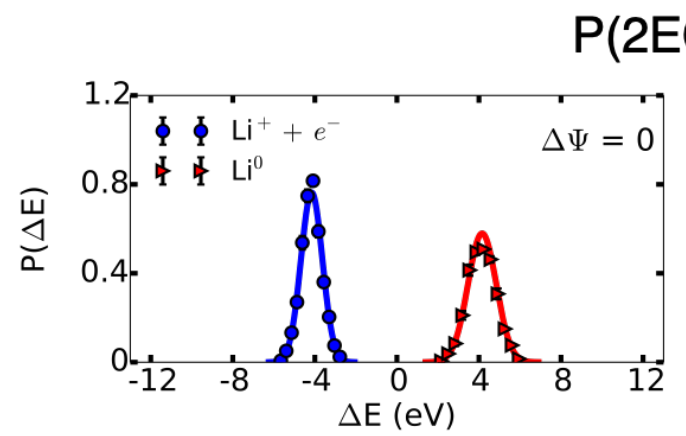

(B)

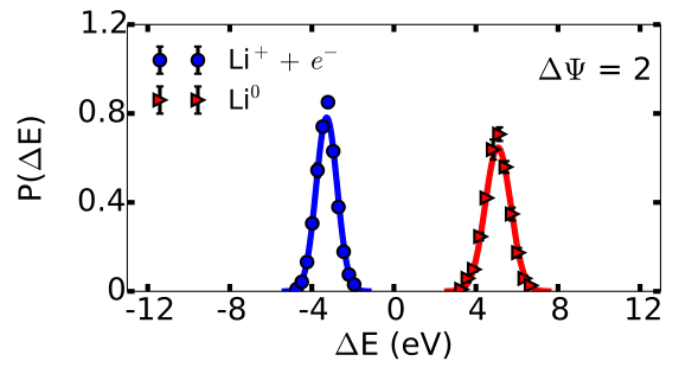

(C)

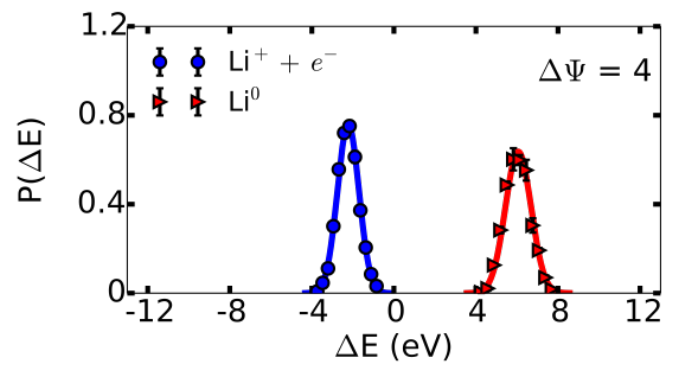

(D)

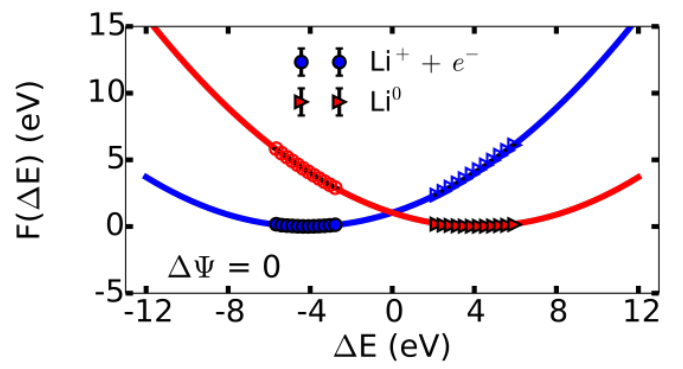

(E)

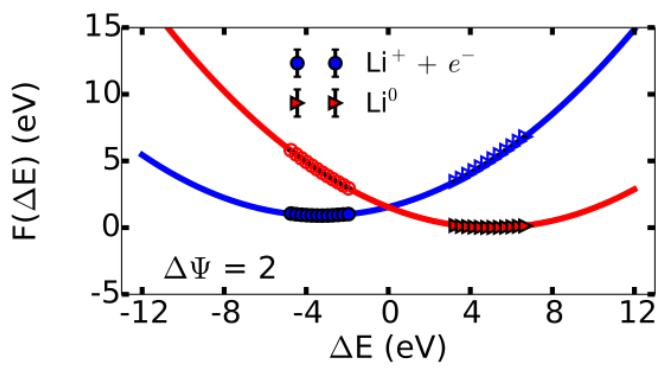

(F)

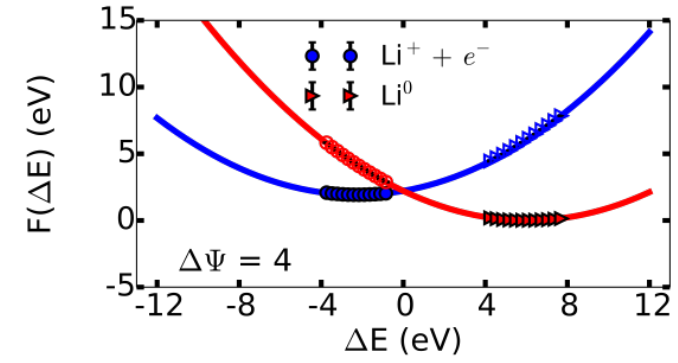

Figure S3: Lithium electroreduction in $\mathrm{P}(2 \mathrm{EO}-\mathrm{MO})$ at the anode interface. (A, B, and $\mathrm{C}$ ) Probability distributions $P(\Delta E)$ of the vertical energy gap $\Delta E$, and $(\mathrm{D}, \mathrm{E}$, and $\mathrm{F}$ ) their associated free energy curves for both the $\mathrm{Li}^{+}$and $\mathrm{Li}^{0}$ in $\mathrm{P}(2 \mathrm{EO}-\mathrm{MO})$. Results are shown for various bias potentials. Solid lines in $(\mathrm{D}, \mathrm{E}$, and $\mathrm{F})$ are parabolic curves using linear-response assumptions.

This material is available free of charge via the Internet at http://pubs.acs.org/. 
G4

(A)

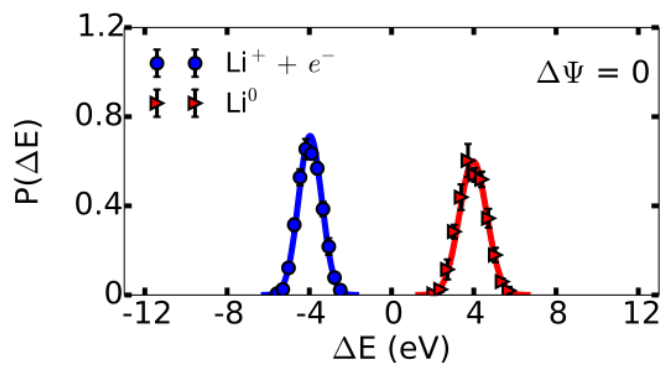

(B)

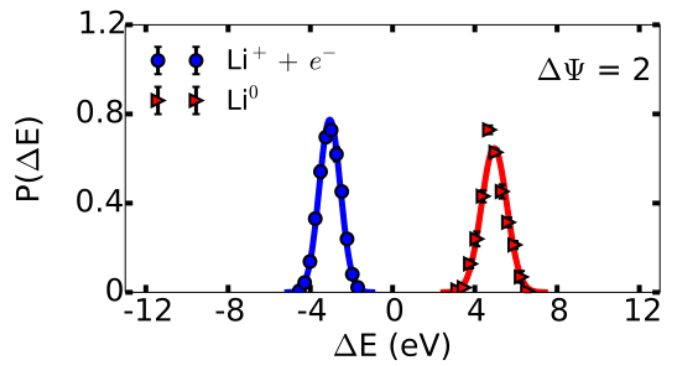

(C)

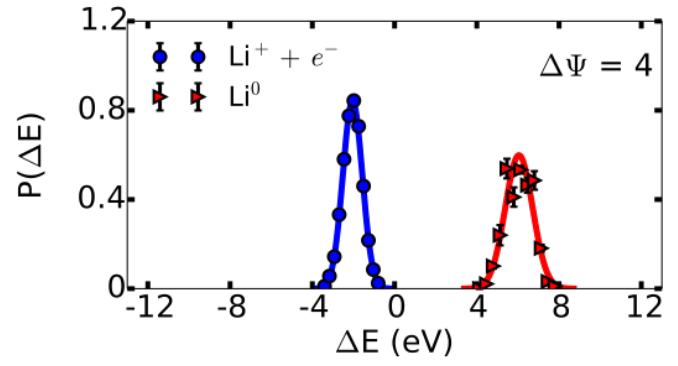

(D)

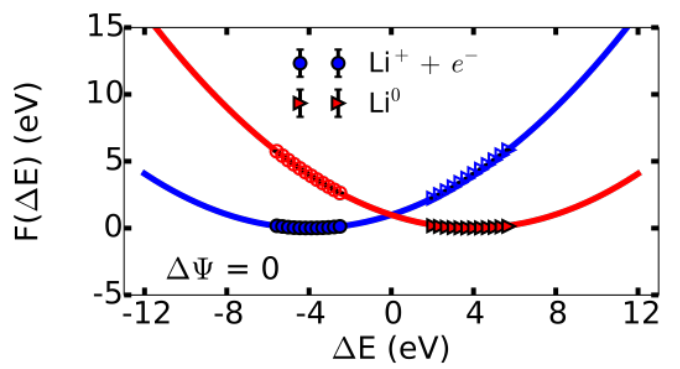

(E)

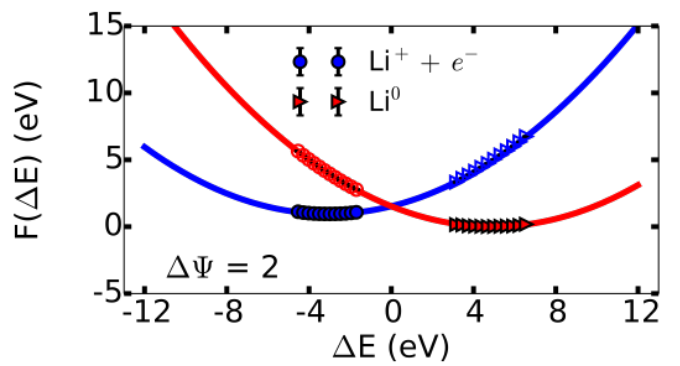

(F)

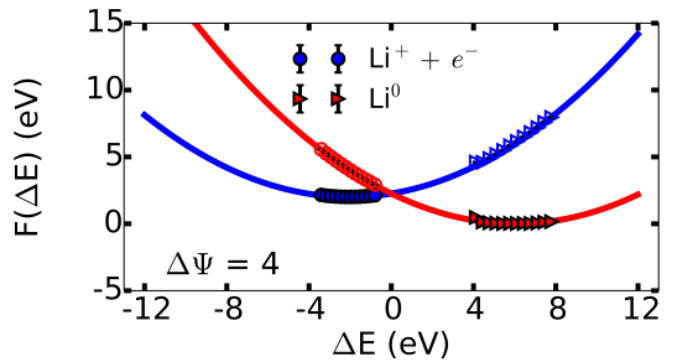

Figure S4: Lithium electroreduction in G4 at the anode interface. (A, B, and C) Probability distributions $P(\Delta E)$ of the vertical energy gap $\Delta E$, and $(\mathrm{D}, \mathrm{E}$, and $\mathrm{F})$ their associated free energy curves for both the $\mathrm{Li}^{+}$and $\mathrm{Li}^{0}$ in G4. Results are shown for various bias potentials. Solid lines in $(\mathrm{D}, \mathrm{E}$, and $\mathrm{F})$ are parabolic curves using linear-response assumptions. 
DME

(A)

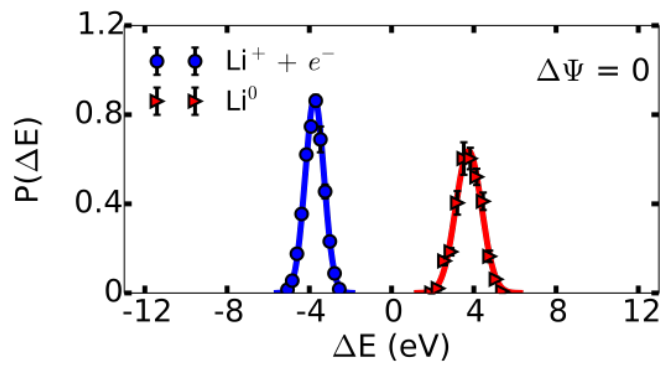

(B)

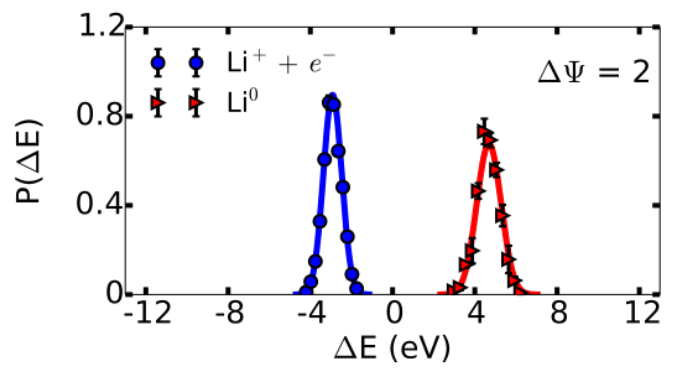

(C)

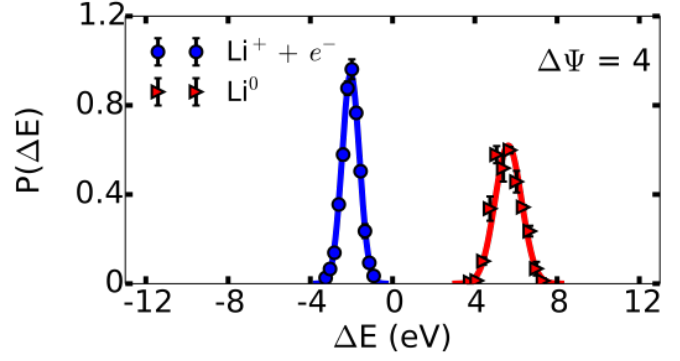

(D)

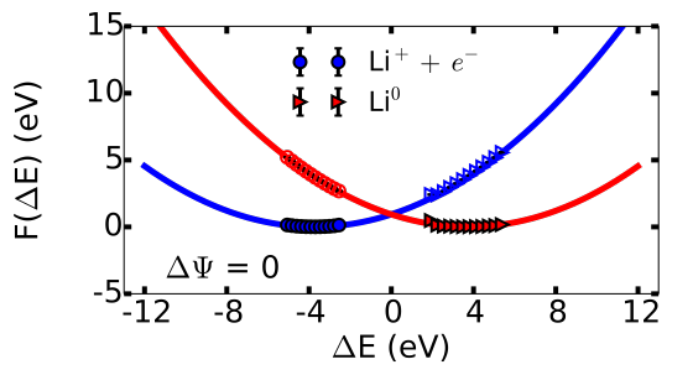

(E)

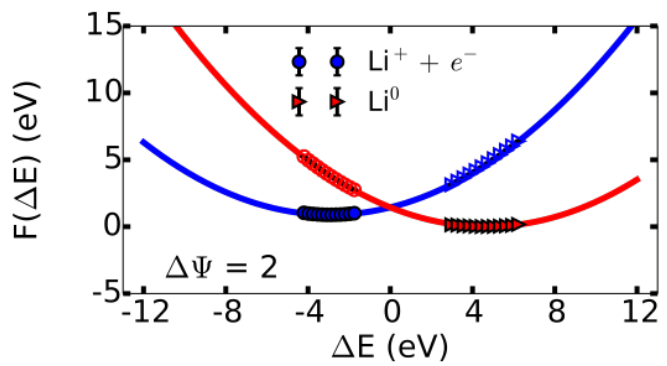

(F)

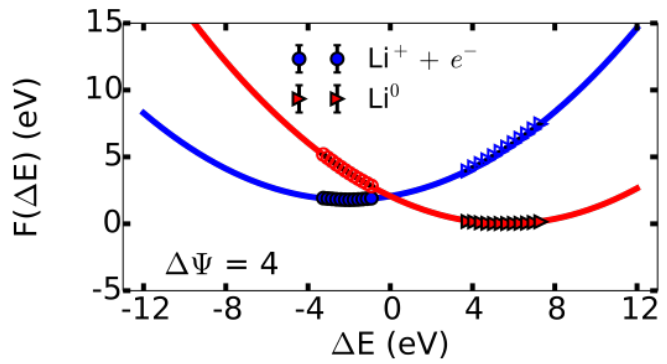

Figure S5: Lithium electroreduction in DME at the anode interface. (A, B, and C) Probability distributions $P(\Delta E)$ of the vertical energy gap $\Delta E$, and $(\mathrm{D}, \mathrm{E}$, and $\mathrm{F}$ ) their associated free energy curves for both the $\mathrm{Li}^{+}$and $\mathrm{Li}^{0}$ in DME. Results are shown for various bias potentials. Solid lines in (D, E, and F) are parabolic curves using linear-response assumptions. 


\section{References}

(1) Reed, S. K.; Madden, P. A.; Papadopoulos, A. The Journal of Chemical Physics 2008, $128,124701$.

(2) Giordano, N. Computational Physics; Prentice Hall, 1997. 
